\title{
Orientation des patients par leur médecin généraliste vers les secteurs hospitaliers publics ou privés en France : étude épidémiologique prospective du réseau Sentinelles.
}

Orientation of patients referred by their GP to the public or private hospital sector in France: a prospective epidemiologic study.

\section{Auteurs}

Paul-Georges Reuter $^{\mathrm{a}, \mathrm{b}}$, Solen Kernéis ${ }^{\mathrm{a}, \mathrm{b}, \mathrm{c}, \mathrm{d}}$, Clément Turbelin ${ }^{\mathrm{a}, \mathrm{b}}$, Cécile Souty ${ }^{\mathrm{a}, \mathrm{b}}$, Christophe Arena ${ }^{\mathrm{a}, \mathrm{b}}$, Gaetan Gavazzi $^{\mathrm{e}, \mathrm{f}}$, Marianne Sarazin ${ }^{\mathrm{a}, \mathrm{b}, \mathrm{g}}$, Thierry Blanchon ${ }^{\mathrm{a}, \mathrm{b}}$, Thomas Hanslik ${ }^{\mathrm{a}, \mathrm{c}, \mathrm{d}}$

\section{Affiliations}

a- INSERM, UMR-S 707, F-75012, Paris, France

b- UPMC Univ Paris 06, UMR-S 707, F-75005, Paris, France

c- Assistance Publique-Hôpitaux de Paris, Service de médecine interne, Hôpital Ambroise-Paré, F92100, Boulogne Billancourt, France

d- Université Versailles-Saint-Quentin-en-Yvelines, F-78000, Versailles, France

e- Clinique Universitaire de médecine Gériatrique, Centre hospitalier Universitaire de Grenoble

f- GREPI, AGIM FRE2405 Université de Grenoble

g- Centre Hospitalier de Firminy, F-42700, Firminy, France

\section{Correspondance}

Thomas Hanslik

Réseau Sentinelles

UMR S 707 Inserm UMPC

Faculté de médecine Pierre et Marie Curie, site Saint Antoine

27 rue Chaligny

75571 Paris cedex 12

Tel : 0144738435

Fax : 0144738454

Email : hanslik@u707.jussieu.fr 


\section{Résumé}

1) Propos : La différence dans les caractéristiques des patients pris en charge à l'hôpital peut générer des différences de coûts entre établissements hospitaliers. Il existe peu de données sur les caractéristiques des patients adressés à l'hôpital par les médecins généralistes libéraux (MG) en France et en particulier sur les facteurs prédictifs de l'orientation des patients vers le secteur privé ou public. Le but de cette étude est d'étudier ces caractéristiques et, en particulier, les facteurs prédictifs de l'orientation des patients vers le secteur privé ou public.

2) Méthodes : Les demandes de recours à l'hospitalisation réalisées par les médecins généralistes du réseau Sentinelles en France métropolitaine ont été recueillies prospectivement entre 2007 et 2009. Les caractéristiques des patients ainsi que les données entourant la demande ont été analysées. La comparaison de la population, en fonction du secteur d'hospitalisation, a été réalisée à l'aide d'une régression logistique.

3) Résultats : Nous avons recueilli 10718 déclarations. II s'agissait de femmes dans $51 \%$ des cas, l'âge médian était de 73 ans et l'hospitalisation n'était programmée que dans $14 \%$ des cas. Les jeunes enfants ainsi que les personnes âgées étaient préférentiellement hospitalisés dans le secteur public $(p<0,001)$. Comparativement aux patients adressés vers le secteur privé, les patients adressés vers le secteur public étaient plus souvent vus dans un contexte d'urgences (OR : 2,3 [2,0-2,8]), par un médecin autre que le médecin traitant (OR : 1,7 [1,4-2,1]) et en dehors du cabinet. Les motifs d'admission étaient différents selon le secteur d'hospitalisation $(p<0,001)$, les malades orientés vers le public présentant une plus grande comorbidité ou complexité diagnostique (sensation d'être malade, évanouissement ou syncope et fièvre par exemple) ou une plus grande dépendance (accident vasculaire cérébral, appareils neurologique et psychiatrique par exemple).

4) Conclusion : Cette étude suggère que les $M G$ orienteraient préférentiellement les patients vers les secteurs d'hospitalisation privé ou public en fonction de critères de gravité, de comorbidité ou de dépendance.

Mots clés : Hospitalisation, Médecin généraliste, Privé, Public, Surveillance 


\section{Abstract}

1) Purpose: In-patients characteristics generate cost differences between hospitals. In France, there are few data on the characteristics on the patients referred to hospitals by their general practitioners (GPs) and none on the predictors of referral to the public or for-profit hospitals. The aim of this study is to analyze those characteristics and the predictors of referral to the public or for-profit hospitals.

2) Methods: We collected, prospectively, the request for hospitalizations made by the GPs of the Sentinelles Network in France, from 2007 to 2009. Patient's characteristics and also the reasons for that request were analyzed. A logistic regression was used to compare the population between local hospitals.

3) Results: 10,718 statements were collected. The median age was 73 years old. Patients were women in $51 \%$ of the cases, and only $14 \%$ of the hospitalizations had been planned.

Hospitalization in the public sector was preferred for young children and the elderly $(p<0,001)$. When compared to the patients referred to the private sector, patients addressed to the public sector were more often seen for emergencies (OR: 2.3 [2.0 - 2.8]), by a doctor different from their referring GP (OR: $1.7[1.4-2.1]$ ) and out of the GP's office. The reasons for hospital admission were different depending on the sector of hospitalization $(p<0.001)$, patients addressed to the public sector hospitals presented with greater comorbidity or more complex diagnosis (for example: feeling ill, fainting or syncope and fever) or a greater disability (for exemple: stroke, neurological and psychiatric diseases).

4) Conclusion: This study suggests that GPs send their patients to the public or for-profit hospitals according to criteria of severity, comorbidity and disability.

Key words: Hospitalization, General practitioner, Private, Public, Surveillance 
Le système hospitalier français est composé d'établissements publics et d'établissements privés (à but lucratif ou sans but lucratif). Ces derniers représentaient, en France, au 31 décembre $2008,65 \%$ des établissements de soins et $36 \%$ de la capacité d'accueil [1]. Le nombre de séjours en hospitalisation complète a été de 12 millions en 2009 [2]. L'activité et les capacités, dans les services Médecine - Chirurgie - Obstétrique, varient selon le cadre juridique des établissements et selon le type d'hospitalisation [2].

Les structures publiques et privées semblent ne pas s'adresser aux mêmes malades. Il a été montré que les hôpitaux publics dispensent plus de soins de nature caritative [3] et prennent en charge plus de pathologies psychiatriques lourdes (considérées comme pathologies peu rentables) [4]. En France, l'Institut de Recherche et Documentation en Economie de la Santé (IRDES) a mis en évidence la large prépondérance du recours au secteur public pour les personnes âgées de plus de 80 ans et pour les prises en charge médicales les plus lourdes. Ce travail a aussi montré une plus grande hétérogénéité des maladies prises en charge dans le secteur public [5].

Le médecin généraliste (MG) occupe une place centrale dans le parcours de soin proposé aux patients entre la ville et l'hôpital. Son implication dans la décision d'hospitalisation est attestée par de multiples études. Par exemple, il a été démontré que le recours à une hospitalisation diminue en cas de suivi régulier par le médecin traitant [6] ou augmente si la demande a été réalisée par un autre médecin que le médecin traitant [7]. II a aussi été démontré qu'une hospitalisation sur dix était décidée par le MG [8] et que $12 \%$ des consultations aux urgences étaient demandées par le médecin traitant [9]. Il existe toutefois peu de données, en France, sur le nombre et les caractéristiques des patients adressés à l'hôpital par le MG et sur son rôle dans l'orientation des patients vers le secteur privé ou public. En particulier, on ne sait pas si les MG orientent les patients vers l'un des deux secteur en tenant compte des caractéristiques propres au malade ou aux circonstance de la consultation ayant aboutit à la décision d'hospitalisation. Une meilleure compréhension du circuit de soin des patients suivis en médecine générale pourrait aider à comprendre les facteurs contribuant à la différence des coûts entre établissements et permettre une meilleure adéquation entre budget alloué et besoins, optimisant ainsi la répartition des rôles dévolus aux différents types de structures hospitalières [5]. Cette étude a pour but de déterminer les caractéristiques des patients orientés vers les secteurs privés ou publics lors du recours à l'hospitalisation, sur la base des données recueillies au cours des trois dernières années par les MG du réseau Sentinelles de l'Inserm.

\section{Méthodes}

Le réseau Sentinelles est un réseau de recherche et de veille en médecine générale en France métropolitaine, créé en 1984, intégré dans l'Unité Mixte de Recherche en Santé UMR S 707 de l'Institut national de la santé et de la recherche médicale (Inserm) et de l'Université Pierre et Marie Curie (UPMC) [10]. Ce système national de recueil d'informations permet l'analyse et la redistribution de données épidémiologiques (recueillies en continu) issues de l'activité des MG de France métropolitaine. Au 1er janvier 2010, 1321 MG étaient inscrits au réseau Sentinelles, soit 2,2\% des médecins généralistes français métropolitains ayant une activité libérale exclusive ou mixte. En 2009, 
un tiers des médecins généralistes Sentinelles (MG Sentinelles) avait participé à l'activité de surveillance continue (soit $0,7 \%$ des MG en France métropolitaine).

Afin d'évaluer la représentativité des MG ayant participé à la surveillance des recours à I'hospitalisation, nous avons comparé les caractéristiques des médecins Sentinelles ayant participé à l'étude à celles des médecins généralistes français [11]. Les variables disponibles étaient le sexe, l'âge, le type d'exercice (groupe ou individuel), la pratique ou non d'un mode d'exercice particulier. Ces informations provenaient d'une part de « Système national inter-régime » de la Caisse nationale de l'assurance maladie des travailleurs salariés, et d'autre part du « baromètre santé médecins généralistes 2009, France » de l'Institut national de prévention et d'éducation pour la santé. Les comparaisons entre les deux groupes ont été réalisées grâce au test de Khi2 pour les variables qualitatives et au test de Student pour la variable « âge ».

Une surveillance nationale des recours à une hospitalisation a été instaurée en 1997. Depuis cette date, les MG déclarent « toute hospitalisation décidée à l'issue d'une consultation ou d'une visite ». L'analyse présentée ici a été effectuée en 2010, elle se basait sur les données recueillies à partir du formulaire déjà existant pour la surveillance systématique des recours à l'hospitalisation. Le format électronique du questionnaire et de la base de données a été modifié au cours de l'année 2007, et nous avons donc limité notre analyse aux données d'hospitalisation transmises entre la 25 ème semaine de 2007 et la dernière semaine de 2009. La répartition des déclarations dans le temps est restée stable tout au long de la période d'étude (courbe d'incidence consultable sur le site Internet du réseau Sentinelles : http://www.sentiweb.fr).

Pour chaque cas rapporté, les MG Sentinelles renseignaient l'âge et le sexe du patient ainsi que le motif d'hospitalisation en texte libre. Ce motif était codé secondairement par un médecin de l'équipe du réseau Sentinelles, selon la classification CISP-2 (Classification Internationale des Soins Primaires) [12]. Ces codes sont composés d'une lettre, correspondant à un appareil, et de deux chiffres spécifiant la rubrique du diagnostic ou du signe clinique motivant la consultation. Le MG Sentinelles précise aussi $1^{\circ}$ ) s'il est le médecin habituel du patient ou non, $2^{\circ}$ ) le lieu de consultation où la décision d'hospitalisation a été prise (domicile, cabinet, maison de retraite ou autre), $3^{\circ}$ ) le contexte d'urgence éventuel (urgence simple, hospitalisation programmée, réanimation ou autre) et $4^{\circ}$ ) le secteur d'hospitalisation (public, privé conventionné, privé non conventionné ou hospitalisation à domicile).

Concernant les variables qualitatives, les résultats sont présentés en nombre d'individus (et/ou proportions) ; quant aux variables quantitatives, elles sont décrites par la médiane (et intervalle Interquartile). Les facteurs prédictifs d'orientation vers le secteur public ou privé conventionné basé sur les caractéristiques des patients et sur les conditions entourant le recours à l'hospitalisation, ont été étudiés par une régression logistique, lors d'une analyse univariée puis multivariée. Lors de cette régression logistique, la variable dépendante était le secteur vers lequel avait été orienté le patient (public ou privé), alors que les autres caractéristiques recueillies (voir supra) constituaient les variables indépendantes. Toutes ces variables ont été prises en compte pour l'analyse multivariée, y compris les motifs d'hospitalisation (définis par l'appareil du code CISP). Les résultats ont été rapportés sous la forme d'odds ratio (OR) par rapport à la classe de référence, avec leurs intervalles de confiance à $95 \%$. Dans l'analyse univariée, les facteurs prédictifs ont été testés par des tests de 
Wilcoxon (variables continues) ou de Fisher (variables qualitatives). Tous les tests sont en formulation bilatérale avec seuil de significativité à 0,05.

Les motifs d'hospitalisation de l'ensemble de la période d'étude ont été analysés par type d'appareil (digestif, cardio-vasculaire, respiratoire, musculo-squelettique...). De plus, les dix codes des diagnostics ou motifs les plus fréquemment rapportés ont été détaillés. Les distributions des motifs d'hospitalisation entre les secteurs d'hospitalisation public et privé conventionné ont été comparées par un test du Chi-deux.

\section{Résultats}

Le recueil des données d'hospitalisation a été effectué par 530 MG Sentinelles durant la période d'étude. Ces MG étaient plus souvent des hommes que l'ensemble des MG français $(p<$ $0,0001)$. En revanche, il n'y avait pas de différence en terme d'âge $(p=0,5)$, de type d'exercice (cabinet de groupe ou pas) $(p=0,6)$ et de mode d'exercice (particulier ou pas) $(p=0,4)$.

Entre la 25ème semaine de 2007 (date de mise à jour du formulaire de description) et la dernière semaine de 2009, 10718 hospitalisations ont été rapportées. Il s'agissait de femmes dans $51 \%$ des cas et l'âge médian était de 73 ans (52 - 83). La distribution des âges montrait une proportion importante des hospitalisations chez les moins de 5 ans et chez les sujets âgés de plus de 70 ans avec un pic chez ceux âgés de 80 à 84 ans (figure 1).

Dans $83 \%$ des cas, le médecin ayant demandé l'hospitalisation était le médecin traitant. La décision d'hospitaliser le patient a été prise au domicile dans $52 \%$ des cas, au cabinet dans $39 \%$ des cas et en maison de retraite dans $6 \%$ des cas. Le contexte était considéré par le médecin comme une « urgence simple » dans $80 \%$ des cas, comme une « hospitalisation programmée » dans $14 \%$ des cas et comme « nécessitant une réanimation » dans $4 \%$ des cas.

La description des motifs d'hospitalisation était disponible pour $68 \%$ des cas rapportés. Les demandes d'hospitalisation étaient en relation avec l'un des trois appareils cardio-vasculaire, digestif et appareil dit « général » dans $64 \%$ des cas. Les $21 \%$ suivants regroupaient les appareils suivants : respiratoire, psychiatrique et musculo-squelettique (figure 2).

Les MG Sentinelles ont adressés $79,1 \%$ des patients vers le secteur public, $20,5 \%$ vers le secteur privé conventionné, $0,2 \%$ vers le secteur privé non conventionné et $0,2 \%$ vers l'hospitalisation à domicile. L'âge médian des patients adressés dans le secteur public était de 73 ans (51 - 83), contre 69 ans $(50$ - 82) dans le secteur privé conventionné $(p<0,001)$. Les résultats de la régression logistique sont présentés dans le tableau 1. Lors de l'analyse multivariée, il n'y avait pas de le sexe des patients n'était pas associé à l'orientation public/privé $(O R=1,0[0,9-1,1])$. Les jeunes enfants étaient préférentiellement hospitalisés dans le secteur public (OR à 6,9 [3,8-14,1]) (tableau 1 et figure 3). Pour les patients de plus de 85 ans, une orientation préférentielle vers le secteur public était retrouvée dans l'analyse univariée ( $O R=1,3[1,2-1,5]$ ) (tableau 1 et figure 3), ce qui n'était pas confirmé après l'analyse multivariée ( $O R=0,9[0,8-1,1])$. Toujours en analyse multivariée, l'orientation vers le secteur public était associée aux caractéristiques suivante : patients vus par un autre médecin que leur médecin habituel ( $O R=1,7[1,4-2,1])$, hors du cabinet de consultation (domicile (OR $=1,6[1,4-1,8])$, maison de retraite $(O R=4,4[3,0-6,6])$, autre $(O R=1,2[0,8-1,9])$ ) ou dans un contexte d'urgence $(\mathrm{OR}=2,3[2,0-2,8])$ (tableau 1). 
La distribution des causes d'hospitalisations variait selon le secteur d'hospitalisation $(p<0,001)$ (figure 4). Les patients adressés pour des causes en rapport avec une atteinte des appareils digestif, musculo-squelettique, urinaire, appareil dit « sexe masculin » et appareil dit « sexe féminin » étaient plus souvent adressés vers le secteur privé. Les dix codes CISP les plus représentés différaient également d'un secteur à l'autre (tableau 2). Ainsi, le code « sensation d'être malade » était la première cause d'envoi vers le secteur public alors qu'elle n'était que la quatrième pour le secteur privé. Par ailleurs, les maladies digestives étaient représentées quatre fois dans le secteur privé (dont la première et la deuxième place) contre deux fois dans le secteur public (la quatrième et la huitième place). Parmi ces 10 codes les plus fréquents, les codes " accident vasculaire cérébral » et « fièvre " n'étaient présents que dans le secteur public, alors que les codes " cardiopathie ischémique avec angor » et « infarctus myocardique aigu » n'étaient présents que dans le secteur privé. Ces dix codes représentaient $34 \%$ de la totalité des demandes d'hospitalisation pour le secteur public et $32 \%$ pour le secteur privé.

\section{Discussion}

Ce travail montre qu'il existe des disparités en termes d'âge, de contexte déclenchant I'hospitalisation et de motif d'hospitalisation selon l'orientation Public/Privé choisie par le MG, indépendamment du motif d'hospitalisation.

Si le très jeune âge apparaît comme un élément prédictif d'orientation vers le secteur public, l'orientation vers un secteur spécifique n'est pas associée à l'âge en lui-même pour les sujets âgés. Les observations pour les moins de 5 ans peuvent être dues au faible nombre de service de Pédiatrie dans le secteur privé. En effet, seulement $4 \%$ des services d'urgences Pédiatrique se trouvent en secteur privé [2]. Dans la population âgée, d'autres facteurs étaient prédictifs de l'orientation, comme la décision du médecin selon qu'il soit le médecin habituel ou non, le lieu de consultation ou le contexte d'urgence. Ainsi, les sujets vus par un autre médecin que le médecin habituel, dans l'urgence et hors du cabinet de consultation ont été davantage adressés vers un hôpital public. A âge égal, les pensionnaires de maison de retraite ont quatre fois plus de chances que les patients vus en consultation d'être orientés vers le public. Ces constatations soulèvent l'hypothèse d'une orientation préférentielle vers le secteur public des patients fragilisés ou nécessitant les soins les plus urgents. En effet, les patients ne venant pas au cabinet de leur médecin sont probablement trop faibles ou trop dépendants pour effectuer ce déplacement, et ceux incapables d'attendre un rendez-vous avec leur médecin habituel ou vus dans un contexte d'urgence ont probablement besoin d'un recours à l'hospitalisation sans délai. Les différences observées entre les causes d'hospitalisation renforcent cette hypothèse, les malades orientés vers le public présentant une plus grande comorbidité ou complexité diagnostique (sensation d'être malade, évanouissement ou syncope et fièvre par exemple) ou une plus grande dépendance (accident vasculaire cérébral, appareils neurologique et psychiatrique par exemple).

Dans $79 \%$ des cas, les médecins du réseau Sentinelles ont adressé leurs patients vers le secteur public. En 2009, ce secteur représentait 57\% du nombre total d'entrées (en hospitalisation complète) et venues (en hospitalisation partielle) sur le territoire français [2]. Cette différence ne peut être expliquée à partir des données recueillies. Plusieurs hypothèses peuvent être formulées pour 
l'expliquer, comme par exemple un recours plus fréquent au secteur public des MG comparativement aux médecins spécialistes ou une particularité des MG du Réseau Sentinelles par rapport à l'ensemble des MG.

En 2009, l'IRDES a rédigé un document de travail portant sur la convergence tarifaire entre les secteurs public et privé en France [5]. Ce travail avait pour objectif d'analyser les justifications économiques de cette convergence. II a mis en évidence la large prépondérance du recours au secteur public des personnes âgées de plus de 80 ans, toutes pathologies confondues, avec une différence plus marquée pour certaines pathologies (pose de prothèse totale de hanche, soins palliatifs ou interventions sur l'intestin ou le colon). Ces différences sont majorées pour les patients de plus de 90 ans présentant une insuffisance cardiaque ou une broncho-pneumopathie. La différence de l'âge médian retrouvée dans notre étude n'est donc pas un résultat isolé. Le travail de l'IRDES souligne également que les prises en charge " lourdes » (selon les critères de l'Outil d'Analyse du Programme de Médicalisation des Systèmes d'Information) sont majoritairement réalisées dans le secteur public, et ce, d'autant plus pour les activités médicales comparativement aux activités chirurgicales. Le document de travail de l'IRDES montre aussi une moins grande hétérogénéité des maladies prises en charge dans le secteur privé : $80 \%$ de l'activité du secteur privé est répartie sur 82 Groupes Homogènes de Malades (GHM), contre 155 GHM dans le secteur public. Les données rapportées dans notre étude vont également dans ce sens. Aux USA également, il a été montré que les hôpitaux à but lucratif étaient plus susceptibles de fournir des services médicaux rentables, alors que les hôpitaux publics étaient plus susceptibles d'offrir des services médicaux peu rentables [13] ou «à visée caritative » [3]. II a aussi été montré que les patients pris en charge dans un hôpital à but lucratif avaient un taux de réadmission dans une autre type de structure (dont l'hôpital public) plus important que les patients pris en charge initialement dans un hôpital public [14].

L'étude que nous rapportons comporte cependant des limites : i) il n'existe pas de suivi des demandes d'hospitalisation effectuées par les médecins Sentinelles et notamment concernant l'orientation finale du patient ; ii) il peut y avoir un biais lié à une sous déclaration des MG Sentinelles de façon générale, voir plus particulièrement sur les hospitalisations programmées ; en ce qui concerne l'analyse comparative des secteurs d'hospitalisation privé et public, il faut prendre en compte la localisation géographique du cabinet médical qui peut être un frein au choix du secteur d'hospitalisation pour le médecin et le patient ; iii) la représentativité du réseau Sentinelles peut être discutée, puisqu'il a été montré que les MG Sentinelles ayant participé à ce recueil de données étaient plus fréquemment des hommes. Toutefois nous nous sommes assurés qu'ils étaient comparables à l'ensemble des MG français pour le type et le mode d'exercice ainsi que pour leur âge [11]. De même, il faut souligner ici les qualités des données utilisées pour notre étude, comme par exemple un nombre élevé de déclarations analysées, la répartition des médecins généralistes Sentinelles sur tout le territoire, le caractère prospectif et standardisé des données recueillies, la mise en œuvre de mesures de contrôle qualité dans les procédures du réseau Sentinelle ou encore le caractère actualisé des données recueillies ; et iv) il est également possible que les MG ayant participé spécifiquement à la surveillance des hospitalisations soient différents des MG participant aux autres surveillance, ce que nous ne pouvons déterminer dans le cadre de cette étude. Toutefois, notre analyse se fonde sur une comparaison des patients en fonction du secteur d'orientation privé ou 
public, recrutés par les mêmes médecins. Elle ne compare pas des patients recrutés par les MG Sentinelles à des patients recrutés par d'autres MG, ce qui limite l'impact des biais de représentativité.

Cette étude prospective nationale, portant sur plus de 10700 déclarations d'hospitalisation, permet d'évaluer le rôle des MG dans le recours à l'hospitalisation. II montre que ce recours est sollicité de manière non programmée dans plus de $80 \%$ des cas et de façon prépondérante vers le secteur hospitalier public. Les différences retrouvées entre les malades adressés vers les secteurs public et privé suggèrent que les MG orienteraient préférentiellement les patients vers l'un ou l'autre de ces secteurs selon des critères de gravité, de comorbidité ou de dépendance, indépendamment du motif d'hospitalisation. Ainsi, les MG auraient recours de façon différentiée aux secteurs d'hospitalisation privé et public, soulignant leur vision du caractère complémentaire mais non superposable de ces structures hospitalières. Ces résultats nécessitent cependant d'être validés par des études complémentaires. Par exemple, une enquête complémentaire notamment qualitative chez les médecins du réseau sentinelles pourrait aider à comprendre les motivations profondes de ces choix d'orientation (plus grand nombre de spécialistes d'organe dans le secteur privé et moins de médecins orientés vers une prise en charge globale, ou tarification à l'acte ne favorisant pas la prise en charge des malades les plus dépendants par exemple). Ces informations sur la répartition des tâches entre les secteurs privé et public sont à prendre en compte à une époque où est évoquée la convergence tarifaire entre les structures hospitalières privées et publiques.

\section{Conflit d'intérêt}

Aucun.

\section{Remerciements}

Les auteurs tiennent à remercier tous les médecins Sentinelles, sans qui cette étude n'aurait pu avoir lieu.

\section{Références}

1. Établissements de santé ayant des capacités d'hospitalisation en 2009 [Internet]. 2011 [cité le 1 novembre 2011]. Consultable sur http://www.insee.fr/fr/themes/tableau.asp?reg_id=0\&ref_id=nattef06116

2. Le panorama des établissements de Santé [Internet]. 2011 [cité le 1 mai 2012]. Consultable sur http://www.drees.sante.gouv.fr/IMG/pdf/etabsante2011.pdf

3. Clement JP, White KR, Valdmanis V. Charity care: do not-for-profits influence for-profits? Med Care Res Rev. 2002 mars;59(1):59-78.

4. Shen JJ, Cochran CR, Moseley CB. From the emergency department to the general hospital: hospital ownership and market factors in the admission of the seriously mentally ill. J Healthc Manag. 2008 août;53(4):268-279; discussion 279-280.

5. Or Z, Renaud T, Com-Ruelle L. Les écarts des coûts hospitaliers sont-ils justifiables ? Rapport de I'Institut de Recherche et Documentation en Economie de la Santé. Paris; 2009.

6. Menec VH, Sirski M, Attawar D, Katz A. Does continuity of care with a family physician reduce hospitalizations among older adults? J Health Serv Res Policy 2006;11:196-201.

7. Fernandes VB, Caldeira AP, Faria AA, Rodrigues Neto JF. Hospitalizations sensitive to primary care as an evaluation indicator for the Family Health Strategy. Rev SaudePublica 2009;43:928-36.

8. Letrilliart $L$, Flahault $A$. Une hospitalisation sur dix est décidée par le médecin généraliste. Réseau Sentinelles. Le quotidien du médecin, 26 Avril 2001. №6906 
9. Eikeland $\mathrm{G}$, Garåsen $\mathrm{H}$, Jacobsen $\mathrm{G}$. Are there alternatives to emergency admissions? Tidsskr Nor Laegeforen 2005;125:2355-7.

10. Websenti.b3e.jussieu.fr [site internet] Rapport annuel 2009 du Réseau Sentinelles. Consultable sur http://websenti.b3e.jussieu.fr/sentiweb/?rub=39

11. Chauvin P, Valleron AJ. Attitude of French general practitioners to the public health surveillance of communicable diseases. Int J Epidemiol. 1995 avr;24(2):435-40.

12. Soler J-K, Okkes I, Wood M, Lamberts $\mathrm{H}$. The coming of age of ICPC: celebrating the $21 \mathrm{st}$ birthday of the International Classification of Primary Care. Family Practice 2008; 25: 312-317.

13. Horwitz JR. Hospitals making profits and providing care: comparing nonprofit, for-profit, and government. Health Affairs 2005;24:790-801.

14. Kind AJH, Bartels C, Mell MW, Mullahy J, Smith M. For-profit hospital status and rehospitalizations at different hospitals: an analysis of Medicare data. Ann. Intern. Med. 2010 déc 7;153(11):718-27.

15. Legrand J. Etude de la représentativité et de la participation des Médecins Sentinelles [Mémoire de DEA]. Paris; 2001. 
Tableau 1. Comparaison des caractéristiques des patients orientés vers les secteurs privés ou publics lors du recours à l'hospitalisation

$\mathrm{N}(\%)$

\begin{tabular}{|c|c|c|c|c|c|}
\hline & $\mathrm{N}$ & $\begin{array}{c}\text { Public } \\
\mathrm{N}=8314\end{array}$ & $\begin{array}{c}\text { Privé } \\
\mathrm{N}=2152\end{array}$ & $\begin{array}{l}\text { OR, analyse univariée } \\
\text { [IC95\%] }\end{array}$ & $\begin{array}{c}\text { OR, analyse multivariée } \\
\text { [IC95\%] }\end{array}$ \\
\hline Sexe du patient adressé en hospitalisation & 10338 & & & & \\
\hline Féminin & & $4231(52)$ & $1073(51)$ & 1 & 1 \\
\hline Masculin & & & & $1,0[0,9-1,1]$ & $1,0[0,9-1,1]$ \\
\hline Age du patient adressé en hospitalisation & 10355 & & & & \\
\hline $0-4$ ans & & $364(4)$ & $13(1)$ & $7,9^{\dagger}[4,7-14,5]$ & $6,9^{\dagger}[3,8-14,1]$ \\
\hline 85 ans et plus & & $1441(18)$ & $301(14)$ & $1,3^{+}[1,2-1,5]$ & $0,9^{+}[0,8-1,1]$ \\
\hline $\begin{array}{l}\text { Hospitalisation à la demande du médecin } \\
\text { habituel }\end{array}$ & 10294 & & & & \\
\hline Oui & & $6630(80)$ & $1869(87)$ & 1 & 1 \\
\hline Non & & $1684(20)$ & $283(13)$ & $1,7[1,5-1,9]$ & $1,7[1,4-2,1]$ \\
\hline Lieu de consultation & 10363 & & & & \\
\hline Cabinet & & $2936(36)$ & $1061(50)$ & 1 & 1 \\
\hline Domicile & & $4435(54)$ & $962(45)$ & $1,7[1,5-1,8]$ & $1,6[1,4-1,8]$ \\
\hline Maison de Retraite & & $601(7)$ & $55(3)$ & $3,9[3,0-5,3]$ & $4,4[3,0-6,6]$ \\
\hline Autre & & $260(3)$ & $53(3)$ & $1,8[1,3-2,4]$ & $1,2[0,8-1,9]$ \\
\hline Contexte & 10394 & & & & \\
\hline Hospitalisation programmée & & $922(11)$ & $543(26)$ & 1 & 1 \\
\hline Urgence simple & & $6814(83)$ & $1488(70)$ & $2,7[2,4-3,0]$ & $2,3[2,0-2,8]$ \\
\hline Réanimation & & $332(4)$ & $64(3)$ & $3,1[2,3-4,1]$ & $2,2[1,5-3,3]$ \\
\hline Autre & & $193(2)$ & $38(2)$ & $3,0[2,1-4,4]$ & $3,4[2,0-5,9]$ \\
\hline
\end{tabular}


Tableau 2. Liste des 10 codes de la Classification Internationale des Soins Primaires les plus fréquents en fonction du secteur d'hospitalisation

\section{N (\%)}

\begin{tabular}{|c|c|c|c|c|c|c|}
\hline \multirow[t]{2}{*}{ Rang } & \multicolumn{3}{|c|}{ Secteur Public } & \multicolumn{3}{|c|}{ Secteur privé } \\
\hline & Code & Nombre & Description & Code & Nombre & Description \\
\hline 1 & A05 & $540(6,5)$ & Sensation d'être malade & D01 & $118(5,5)$ & $\begin{array}{c}\text { Douleur ou crampes } \\
\text { abdominales } \\
\text { généralisées }\end{array}$ \\
\hline 2 & K77 & $406(4,9)$ & Décompensation cardiaque & D88 & $115(5,3)$ & Appendicite \\
\hline 3 & K90 & $357(4,3)$ & accident vasculaire cérébral & K77 & $103(4,8)$ & $\begin{array}{l}\text { Décompensation } \\
\text { cardiaque }\end{array}$ \\
\hline 4 & D01 & $323(3,9)$ & $\begin{array}{c}\text { Douleur ou crampes } \\
\text { abdominales généralisées }\end{array}$ & A05 & $70(3,3)$ & Sensation d'être malade \\
\hline 5 & R02 & $230(2,8)$ & Sibilance & D98 & $68(3,2)$ & $\begin{array}{l}\text { Cholécystite, } \\
\text { cholélithiase }\end{array}$ \\
\hline 6 & $\mathrm{~A} 03$ & $209(2,5)$ & Fièvre & R02 & $49(2,3)$ & Sibilance \\
\hline 7 & A06 & $206(2,5)$ & Evanouissement ou syncope & K74 & $44(2,0)$ & $\begin{array}{c}\text { Cardiopathie ischémique } \\
\text { avec angor }\end{array}$ \\
\hline 8 & D88 & $191(2,3)$ & Appendicite & D99 & $42(2,0)$ & $\begin{array}{l}\text { Autre maladie du } \\
\text { système digestif }\end{array}$ \\
\hline 9 & A80 & $184(2,2)$ & $\begin{array}{c}\text { Traumatisme ou lésion } \\
\text { traumatique NCA }\end{array}$ & A80 & $40(1,9)$ & $\begin{array}{c}\text { Traumatisme ou lésion } \\
\text { traumatique NCA }\end{array}$ \\
\hline 10 & R81 & $163(2,0)$ & Pneumonie & K75 & $37(1,7)$ & $\begin{array}{l}\text { Infarctus myocardique } \\
\text { aigu }\end{array}$ \\
\hline Total & & $8304(100)$ & & & $2150(100)$ & \\
\hline
\end{tabular}


Figure 1. Distribution des cas hospitalisés à la demande des médecins Sentinelles décrits par tranche d'âges

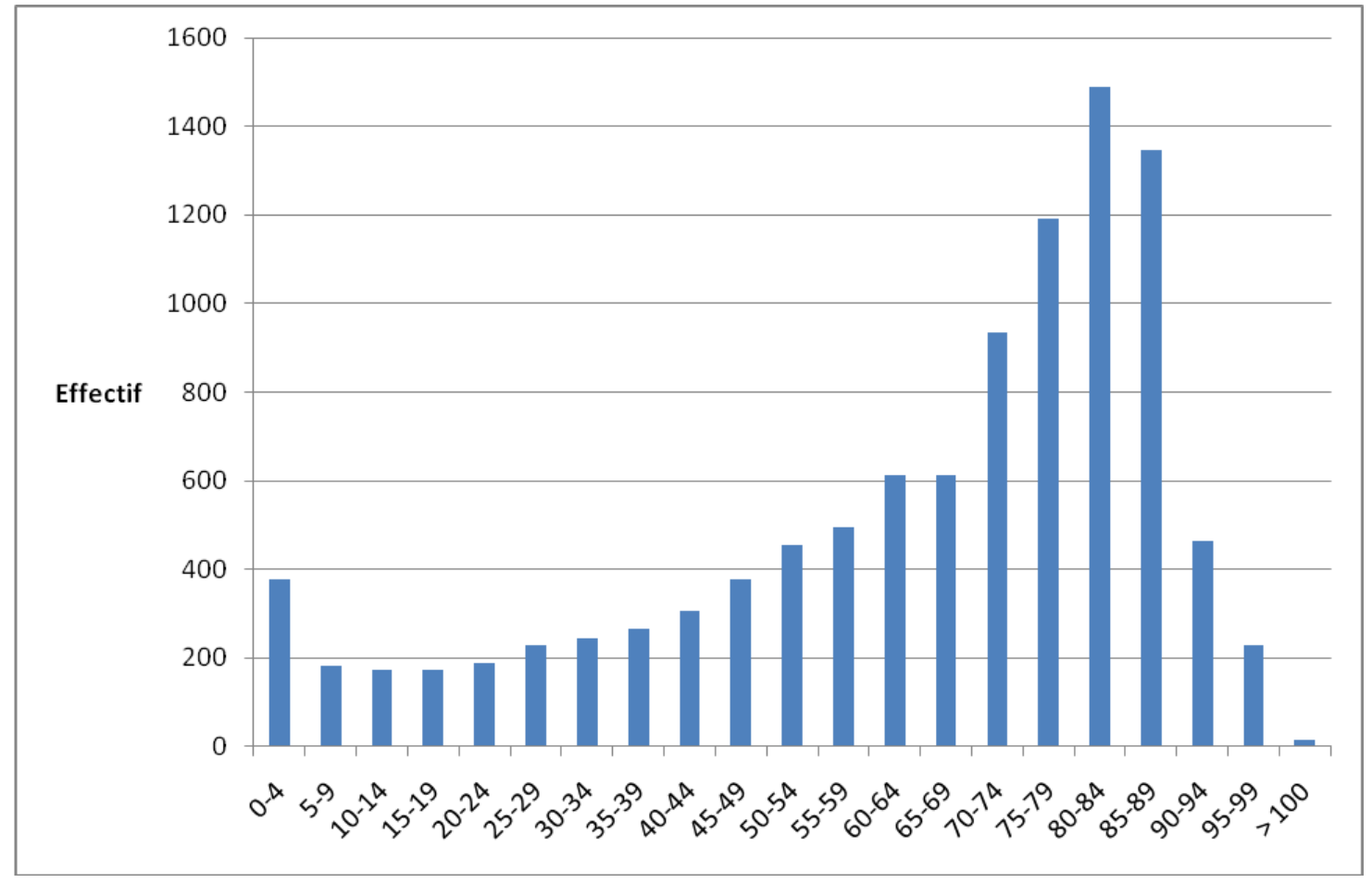


Figure 2. Répartition par appareil des codes de la Classification Internationale des Soins Primaires dans l'ensemble des motifs d'hospitalisation demandée par les médecins Sentinelles

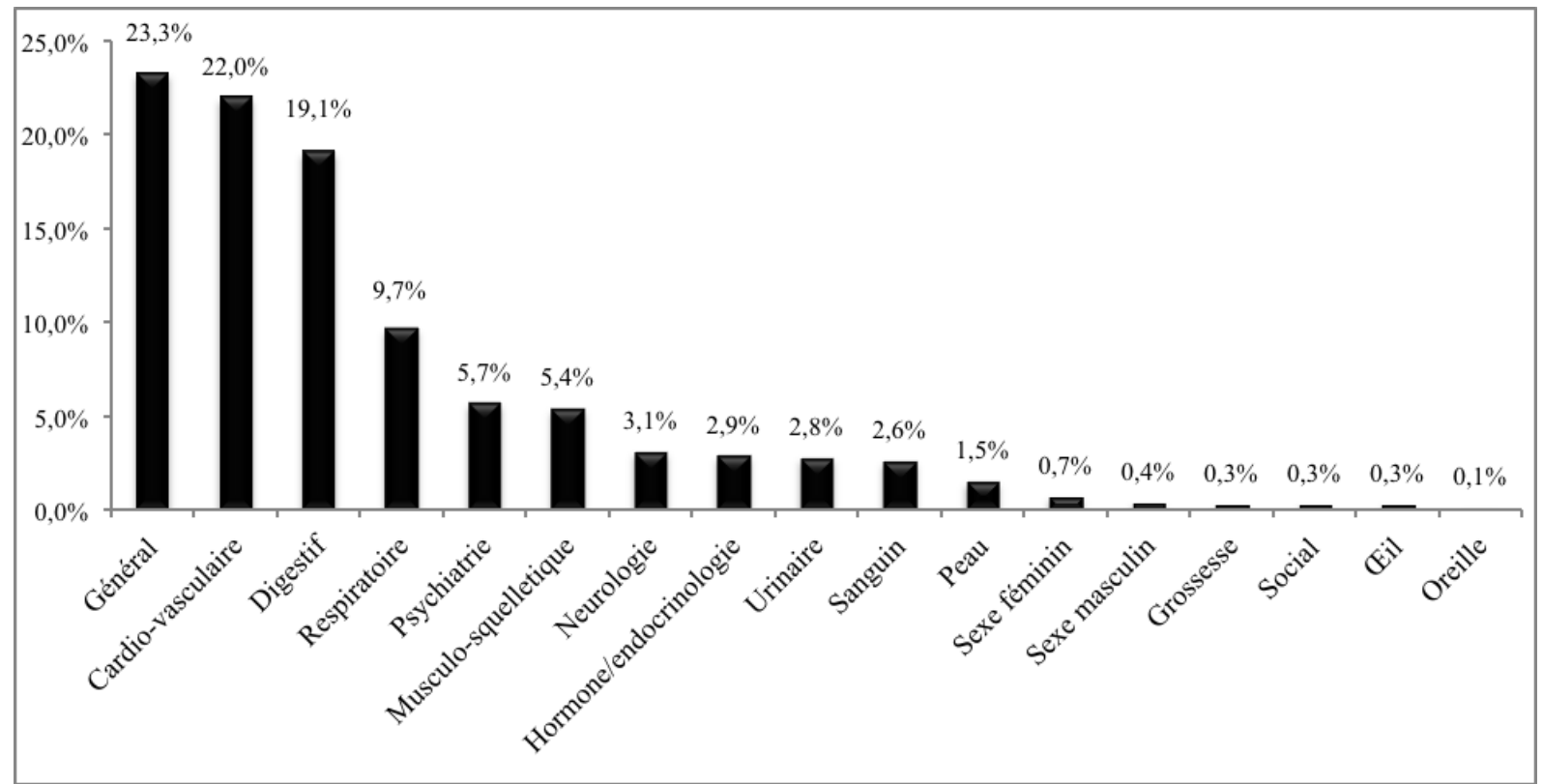


Figure 3. Répartition des hospitalisations demandées par les médecins Sentinelles par tranches d'âges et par secteur, entre 2007s25 et 2009s53

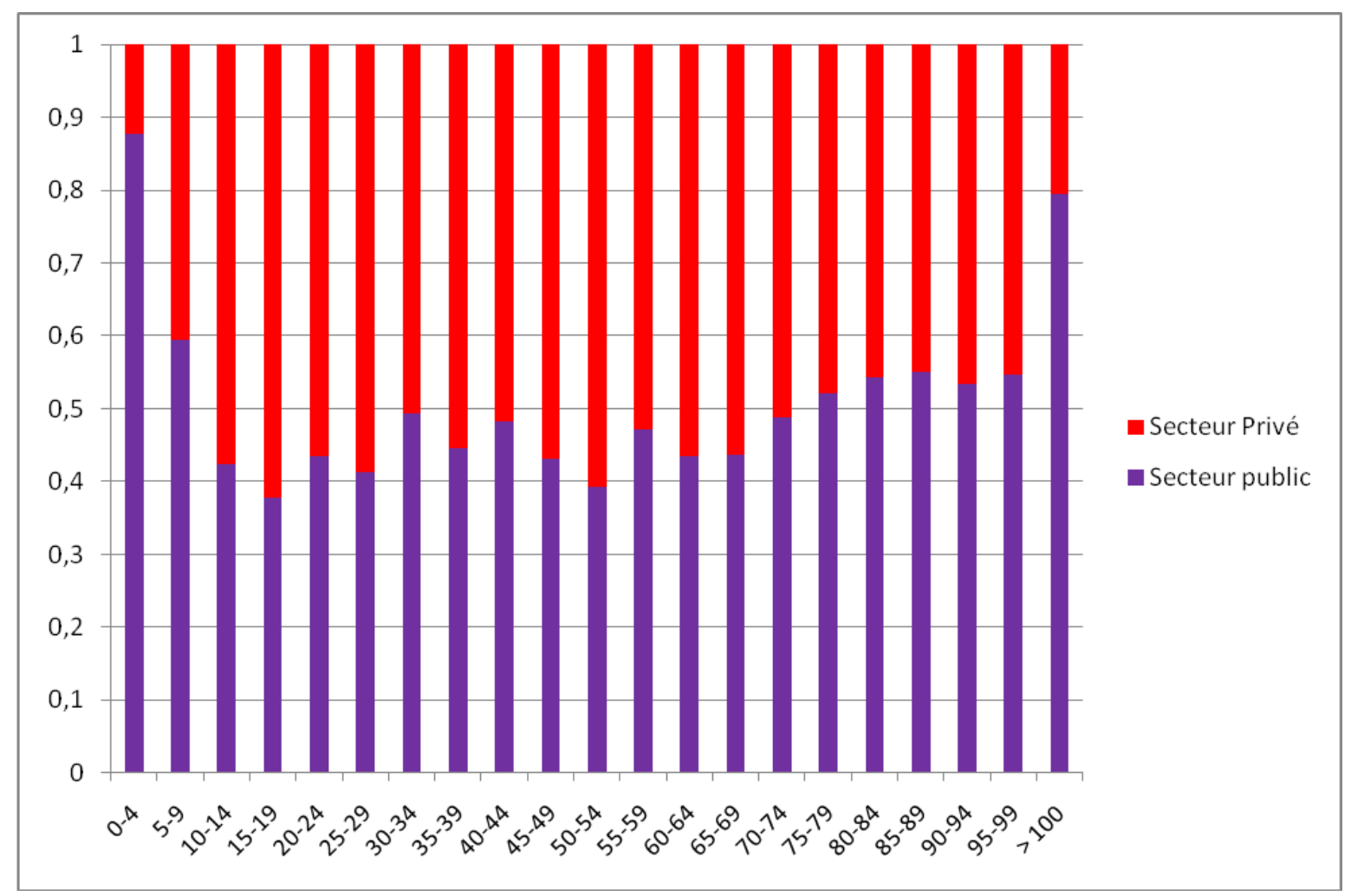


Figure 4. Répartition par appareil des codes de la Classification Internationale des Soins Primaires dans l'ensemble des motifs d'hospitalisation demandée par les médecins Sentinelles, en fonction du secteur d'hospitalisation

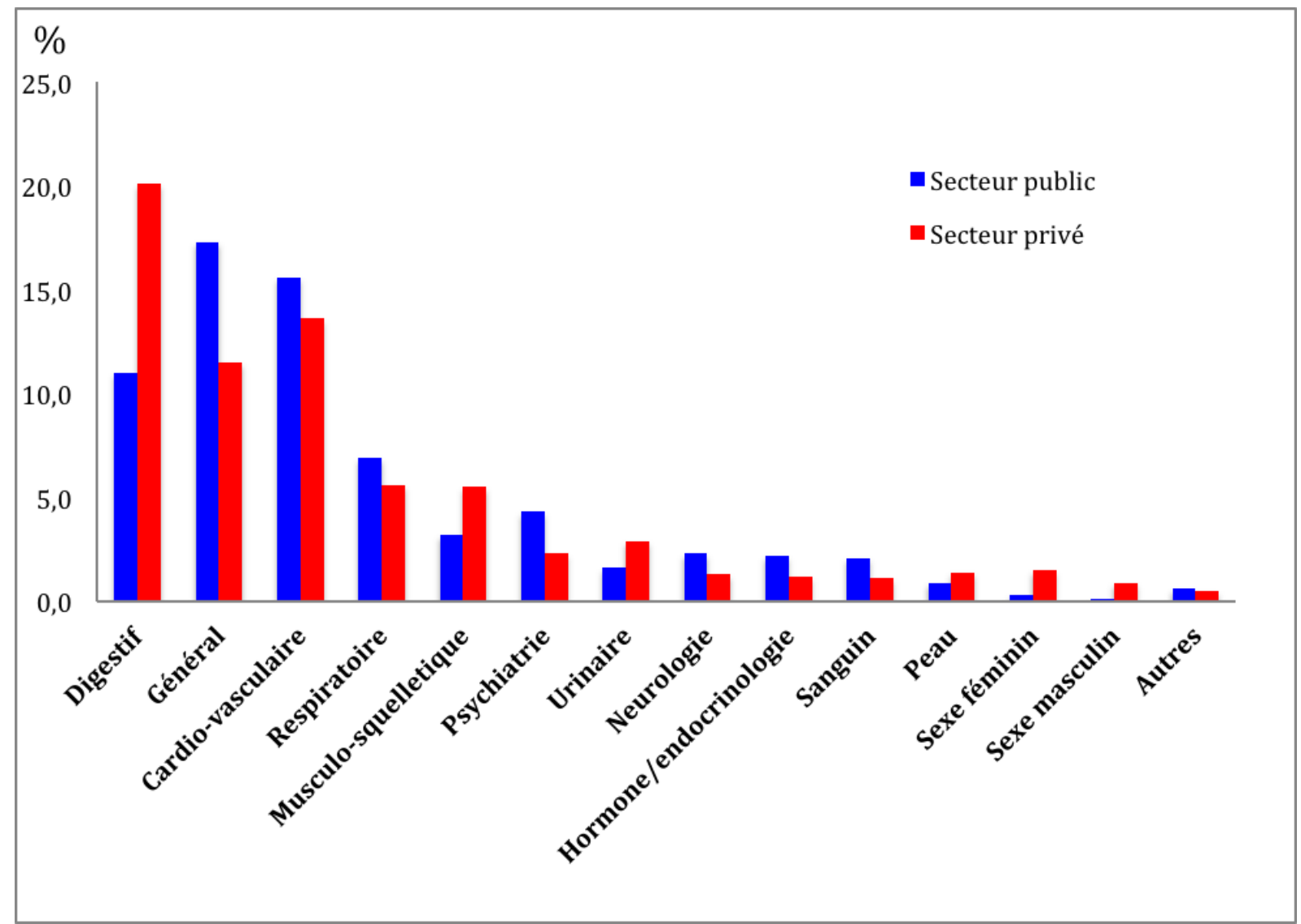

